\title{
An extension of the Krein-`̌smulian Theorem
}

Antonio S. Granero

\begin{abstract}
Let $X$ be a Banach space, $u \in X^{* *}$ and $K, Z$ two subsets of $X^{* *}$. Denote by $d(u, Z)$ and $d(K, Z)$ the distances to $Z$ from the point $u$ and from the subset $K$ respectively. The Krein-Šmulian Theorem asserts that the closed convex hull of a weakly compact subset of a Banach space is weakly compact; in other words, every $\mathrm{w}^{*}$-compact subset $K \subset X^{* *}$ such that $d(K, X)=0$ satisfies $d\left(\overline{\mathrm{CO}}^{w^{*}}(K), X\right)=0$.

We extend this result in the following way: if $Z \subset X$ is a closed subspace of $X$ and $K \subset X^{* *}$ is a $\mathrm{w}^{*}$-compact subset of $X^{* *}$, then

$$
d\left(\overline{\mathrm{co}}^{w^{*}}(K), Z\right) \leq 5 d(K, Z) .
$$

Moreover, if $Z \cap K$ is $\mathrm{w}^{*}$-dense in $K$, then $d\left(\overline{\mathrm{co}}^{w^{*}}(K), Z\right) \leq 2 d(K, Z)$. However, the equality $d(K, X)=d\left(\overline{\mathrm{co}}^{w^{*}}(K), X\right)$ holds in many cases, for instance, if $\ell_{1} \nsubseteq X^{*}$, if $X$ has $\mathrm{w}^{*}$-angelic dual unit ball (for example, if $X$ is WCG or WLD), if $X=\ell_{1}(I)$, if $K$ is fragmented by the norm of $X^{* *}$, etc. We also construct under $C H$ a w*-compact subset $K \subset B\left(X^{* *}\right)$ such that $K \cap X$ is $\mathrm{w}^{*}$-dense in $K, d(K, X)=\frac{1}{2}$ and $d\left(\overline{\mathrm{co}}^{w^{*}}(K), X\right)=1$.
\end{abstract}

\section{Introduction}

If $X$ is a Banach space, let $B(X)$ and $S(X)$ be the closed unit ball and unit sphere of $X$, respectively, and $X^{*}$ its topological dual. If $u \in X^{* *}$ and $K, Z$ are two subsets of $X^{* *}$, let $d(u, Z)=\inf \{\|u-z\|: z \in Z\}$ be the distance to $Z$ from $u, d(K, Z)=\sup \{d(k, Z): k \in K\}$ the distance to $Z$ from $K$, $\operatorname{co}(K)$ the convex hull of $K, \overline{\mathrm{co}}(K)$ the norm-closure of $\operatorname{co}(K)$ and $\overline{\mathrm{co}}^{w^{*}}(K)$ the $\mathrm{w}^{*}$-closure of $\operatorname{co}(K)$.

2000 Mathematics Subject Classification: 46B20, 46B26.

Keywords: Krein-Šmulian Theorem, Banach spaces, compact sets. 
This paper is devoted to investigate the connection between the distances $d\left(\overline{\mathrm{co}}^{w^{*}}(K), Z\right)$ and $d(K, Z)$, when $Z \subset X^{* *}$ is a subspace of $X$ (in particular, when $Z=X)$ and $K$ is a $\mathrm{w}^{*}$-compact subset of $X^{* *}$. There exist some facts that suggest that the distance $d\left(\overline{\mathrm{co}}^{w^{*}}(K), Z\right)$ is controlled by the distance $d(K, Z)$. Indeed, on the one hand, we have the classical Theorem of KreinŠmulian (see [5, p. 51]). Using the terminology of distances, this Theorem asserts the following: if $X$ is a Banach space, every $\mathrm{w}^{*}$-compact subset $K \subset X^{* *}$ with $d(K, X)=0$ (that is, $K \subset X$ is a weakly compact subset of $X$ ) satisfies $d\left(\overline{\mathrm{co}} w^{*}(K), X\right)=0$ (that is, the closed convex hull $\overline{\mathrm{co}}(K)$ of $K$ in $X$ is weakly compact).

On the other hand, if the dual $X^{*}$ of the Banach space $X$ does not contain a copy of $\ell_{1}$, it is very easy to prove that $d(K, Z)=d\left(\overline{\mathrm{co}} w^{*}(K), Z\right)$ for every $\mathrm{w}^{*}$-compact subset $K \subset X^{* *}$ of $X^{* *}$ and every subspace $Z \subset X^{* *}$. Indeed, in this case $\overline{\mathrm{co}}(K)=\overline{\mathrm{co}} w^{*}(K)$ (see [9]). So, as $d(\operatorname{co}(K), Z)=d(K, Z)$ (this follows from the fact that the function $\varphi(u):=d(u, Z), \forall u \in X^{* *}$, is convex when $Z \subset X^{* *}$ is a convex subset of $\left.X^{* *}\right)$, we easily obtain that $d(K, Z)=d\left(\overline{\mathrm{co}}^{w^{*}}(K), Z\right)$.

In view of these facts, one is inclined to conjecture that $d(K, X)=$ $d\left(\overline{\mathrm{co}} w^{*}(K), X\right)$ for every $\mathrm{w}^{*}$-compact subset $K \subset X^{* *}$ and every Banach space $X$. Unfortunately, assuming the Continuum Hypothesis (for short, $\mathrm{CH}$ ), this is not true because of the following result we will prove here.

Theorem 1 Under $C H$, if $X=\ell_{\infty}^{c}\left(\omega^{+}\right)$(= subspace of the elements $f \in$ $\ell_{\infty}\left(\omega^{+}\right)$with countable support $)$, there exists a $w^{*}$-compact subset $H \subset B\left(X^{* *}\right)$ such that $d(H, X)=1 / 2, H \cap X$ is $w^{*}$-dense in $H$ and $d\left(\overline{c o} w^{*}(H), X\right)=1$.

However, there exist many Banach spaces $X$ for which the equality $d(K, X)=d\left(\overline{\mathrm{co}}^{w^{*}}(K), X\right)$ holds, for every $\mathrm{w}^{*}$-compact subset $K \subset X^{* *}$, for example, the class of Banach spaces with property $J$.

Definition $2 A$ Banach space $X$ has property $J$ (for short, $X \in J$ ) if for every $z \in B\left(X^{* *}\right) \backslash X$ and for every number $b \in \mathbb{R}$ with $0<b<d(z, X)$, there exists a sequence $\left\{x_{n}^{*}\right\}_{n \geq 1} \subset \mathfrak{S}\left(B\left(X^{*}\right), z, b\right):=\left\{u \in B\left(X^{*}\right): z(u) \geq b\right\}$ such that $x_{n}^{*} \stackrel{w^{*}}{\rightarrow} 0$.

For this class of Banach spaces with property $J$ we prove the following result.

Theorem 3 Let $X$ be a Banach space such that $X \in J$. Then for every $w^{*}$-compact subset $K \subset X^{* *}$ we have $d(K, X)=d\left(\overline{c o}^{*}(K), X\right)$.

In the following corollary we state that many Banach spaces have property $J$ and, so, satisfy Theorem 3 . Recall that, for a Banach $X$, the dual unit ball $\left(B\left(X^{*}\right), w^{*}\right)$ is angelic in the $\mathrm{w}^{*}$-topology if, for every subset $A \subset B\left(X^{*}\right)$ and every $z \in \bar{A}^{w^{*}}$, there exists a sequence $\left\{a_{n}\right\}_{n \geq 1} \subset A$ such that $a_{n} \stackrel{w^{*}}{\rightarrow} z$. 
Corollary 4 If $X$ is a Banach space such that $\left(B\left(X^{*}\right), w^{*}\right)$ is angelic (for example, if $X$ is weakly compactly generated (for short, WCG) or weakly Lindelöf determined (for short, WLD)), then $X \in J$ and, so, for every $w^{*}$-compact subset $K \subset X^{* *}$ we have $d(K, X)=d\left(\overline{c o}^{w^{*}}(K), X\right)$.

Although the equality $d(K, X)=d\left(\overline{\mathrm{co}} w^{*}(K), X\right)$ does not hold in general, we can ask whether there exists a universal constant $1 \leq M<\infty$ such that $d\left(\overline{\mathrm{co}}^{w^{*}}(K), X\right) \leq M d(K, X)$ for every Banach space $X$ and every $\mathrm{w}^{*}$-compact subset $K \subset X^{* *}$.

The answer to this question is affirmative. We prove the following result, which extends the Krein-Šmulian Theorem.

Theorem 5 If $X$ is a Banach space, $Z \subset X$ a closed subspace of $X$ and $K \subset X^{* *}$ a $w^{*}$-compact subset, then $d\left(\overline{c o}^{w^{*}}(K), Z\right) \leq 5 d(K, Z)$.

When $K \cap Z$ is $\mathrm{w}^{*}$-dense in $K$, the argument used in Theorem 5 gives the following result.

Theorem 6 Let $X$ be a Banach space, $Z \subset X$ a closed subspace and $K \subset$ $X^{* *}$ a $w^{*}$-compact subset. If $Z \cap K$ is $w^{*}$-dense in $K$, then $d\left(\overline{c o}^{w^{*}}(K), Z\right) \leq$ $2 d(K, Z)$.

Finally, we also obtain the following result.

Theorem 7 Let $I$ be an infinite set and $X=\ell_{1}(I)$. Then for every $w^{*}-$ compact subset $K \subset X^{* *}$ we have $d\left(\overline{c o} w^{*}(K), X\right)=d(K, X)$.

A version of the problem we study here was considered (independently) by M. Fabian, P. Hájek, V. Montesinos and V. Zizler in [7]. They study the class of $\mathrm{w}^{*}$-compact subsets $K \subset X^{* *}$ such that $K \cap X$ is $\mathrm{w}^{*}$-dense in $K$. Instead of distances, they deal with the notion of $\epsilon$-weakly relatively compact subsets of $X$ (for short, $\epsilon$-WRK) introduced in [8]. A bounded subset $H$ of $X$ is said to be $\epsilon$-WRK, for some $\epsilon>0$, if $\bar{H}^{w^{*}} \subset X+\epsilon B\left(X^{* *}\right)$, that is, if $d\left(\bar{H}^{w^{*}}, X\right) \leq \epsilon$. Using arguments based on the techniques of double limit due to Grothendieck and Pták, they prove that the constant $M=2$ holds for this category of $\mathrm{w}^{*}$-compact subsets $K \subset X^{* *}$ such that $K \cap X$ is $\mathrm{w}^{*}$-dense in $K$. More precisely, they prove the following beautiful result.

Theorem ([7]) Let $X$ be a Banach space and $H \subset X$ a bounded subset of $X$. Assume that $H$ is $\epsilon$-WRK for some $\epsilon>0$. Then the convex hull co $(H)$ is $2 \epsilon$-WRK. Moreover, if $\left(B\left(X^{*}\right), w^{*}\right)$ is angelic, or $X^{*}$ does not contain a copy of $\ell_{1}$, then $\operatorname{co}(H)$ is $\epsilon-W R K$.

Observe that the Theorem of Krein-Šmulian follows from this result when $\epsilon=0$. 


\section{Proofs of the results}

Let us introduce some notation and terminology (see [1], [4], [6], [11]). $|A|$ denotes the cardinality of a set $A, \omega^{+}$the first uncountable ordinal, $\aleph_{1}$ the first uncountable cardinal and $C H$ the continuum hypothesis. A Hausdorff compact space $K$ is said to have property $(M)$ if every Radon Borel measure $\mu$ on $K$ has separable support $\operatorname{supp}(\mu)$. If $K$ is a convex compact subset of some locally convex linear space $X$ and $\mu$ is a Radon Borel probability measure on $K, r(\mu)$ denotes the barycentre of $\mu$. Recall (see [3]) that $r(\mu) \in K$ and that $r(\mu)$ satisfies $x^{*}(r(\mu))=\int_{K} x^{*}(k) d \mu$ for every $x^{*} \in X^{*}$.

If $X$ is a Banach space, let $X^{\perp}=\left\{z \in X^{* * *}:\langle z, x\rangle=0, \forall x \in X\right\}$ denote the subspace of $X^{* * *}$ orthogonal to $X$. If $Y \subset X$ is a subspace of $X$, let $Y^{\perp}\left(X^{*}\right)=\left\{z \in X^{*}:\langle z, y\rangle=0, \forall y \in Y\right\}$ be the subspace of $X^{*}$ orthogonal to $Y, Y^{\perp}\left(X^{* * *}\right)=\left\{z \in X^{* * *}:\langle z, y\rangle=0, \forall y \in Y\right\}$, etc. So, $X^{\perp}=X^{\perp}\left(X^{* * *}\right)$. Recall that, if $u \in X$ (resp., $\left.u \in X^{* *}\right)$, then $d(u, Y)=\sup \left\{\langle z, u\rangle: z \in B\left(Y^{\perp}\left(X^{*}\right)\right)\right\}$ (resp., $d(u, Y)=\sup \{\langle z, u\rangle: z \in$ $\left.\left.B\left(Y^{\perp}\left(X^{* * *}\right)\right)\right\}\right)$. If $A \subset X$ is a subset of $X,[A]$ denotes the linear span of $A$.

Let $I$ be an infinite set with the discrete topology. Then:

(0) We use the symbol $\ell_{\infty}(I)$ to denote the Banach space of all $f=$ $(f(i))_{i \in I} \in \mathbb{R}^{I}$ with supremum norm finite $\|f\|:=\sup \{|f(i)|: i \in$ $I\}<\infty$. The symbol $c_{0}(I)$ means its subspace consisting from $f=$ $(f(i))_{i \in I} \in \ell_{\infty}(I)$ such that the set $\{i \in I:|f(i)|>\epsilon\}$ is finite for all $\epsilon>0$.

(1) If $f \in \ell_{\infty}(I), \operatorname{supp}(f)=\{i \in I: f(i) \neq 0\}$ will be the support of $f$ and

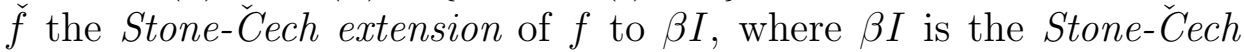
compactification of $I$.

(2) Let $c I=\cup\left\{\bar{A}^{\beta I}: A \subset I, A\right.$ countable $\}$ and $\ell_{\infty}^{c}(I)=\left\{f \in \ell_{\infty}(I)\right.$ : $\operatorname{supp}(f)$ countable $\}$. Observe that $c I$ is an open subset of $\beta I$ and that, if $f \in \ell_{\infty}(I)$, then $f \in \ell_{\infty}^{c}(I)$ if and only if $\check{f}_{\mid \beta I \backslash c I}=0$.

(3) Let $\Sigma\left(\{0,1\}^{I}\right)=\left\{x \in\{0,1\}^{I}: \operatorname{supp}(x)\right.$ countable $\}$ and $\Sigma\left([-1,1]^{I}\right)=$ $\left\{x \in[-1,1]^{I}: \operatorname{supp}(x)\right.$ countable $\}$.

(4) Recall that a compact space is said to be a Corson space if it is homeomorphic to some compact subset of $\Sigma\left([-1,1]^{I}\right)$.

Proof of Theorem 1. We use a modification of the Argyros-Mercourakis-Negrepontis Corson compact space without property $(M)$ [1, p. 219]. In the following we adopt the notation and terminology of [1, p. 219]. Let $\Omega$ be the space of Erdös, that is, the Stone space of the quotient algebra $M_{\lambda} / N_{\lambda}$, where $\lambda$ is the Lebesgue measure on $[0,1], M_{\lambda}$ is the algebra of $\lambda$ measurable subsets of $[0,1]$ and $N_{\lambda}$ is the ideal of $\lambda$-null subsets of $[0,1]$. $\Omega$ is 
a compact extremely disconnected space (because $M_{\lambda} / N_{\lambda}$ is complete) and there exists a strictly positive regular Borel normal probability measure $\tilde{\lambda}$ on $\Omega$, determined by the condition $\tilde{\lambda}(V)=\lambda(U), V$ being any clopen subset of $\Omega$ and $U$ a $\lambda$-measurable subset of $[0,1]$ such that $V=U+N_{\lambda}$.

Now we proceed as in $[1,3.11$ Lemma] with small changes. Write $[0,1]=$ $\left\{x_{\xi}: \xi<\omega^{+}\right\}$and let $\left\{K_{\xi}: \xi<\omega^{+}\right\}$be the well-ordered class of compact subsets of $[0,1]$ with strictly positive Lebesgue measure. For each $\xi<\omega^{+}$ we choose a compact subset $U_{\xi} \subset[0,1]$ such that:

(a) $U_{\xi} \subset\left\{x_{\rho}: \xi<\rho<\omega^{+}\right\} \cap K_{\xi}$.

(b) If $\lambda\left(K_{\xi}\right)=1$, then $U_{\xi}$ satisfies the condition $\lambda\left(U_{\xi}\right)>0$. If $\lambda\left(K_{\xi}\right)<1$, $U_{\xi}$ satisfies the condition $\lambda\left(K_{\xi}\right)-\left(1-\lambda\left(K_{\xi}\right)\right)<\lambda\left(U_{\xi}\right) \leq \lambda\left(K_{\xi}\right)$.

Let $V_{\xi}$ be the clopen subset of $\Omega$ corresponding to $U_{\xi}$. Then $\left\{V_{\xi}: \xi<\omega^{+}\right\}$is a pseudobase of $\Omega$ that witnesses the failure of the property caliber $\omega^{+}$, that is, if $A \subset \omega^{+}$and $|A|=\aleph_{1}$, then $\cap_{\xi \in A} V_{\xi}=\emptyset$. Moreover, (b) automatically implies that $\left|\left\{\xi<\omega^{+}: \lambda\left(U_{\xi}\right)>t\right\}\right|=\aleph_{1}$ for every $0<t<1$, whence $\left|\left\{\xi<\omega^{+}: \tilde{\lambda}\left(V_{\xi}\right)>t\right\}\right|=\aleph_{1}$ for every $0<t<1$.

Consider $\mathcal{A}=\left\{A \subset \omega^{+}: \cap_{\xi \in A} V_{\xi} \neq \emptyset\right\}$. Clearly, $\mathcal{A}$ is an adequate family (see $[11$, p. 1116]) such that every element of $\mathcal{A}$ is a countable subset of $\omega^{+}$. Moreover, there are elements $A \in \mathcal{A}$ with $|A|=\aleph_{0}$. Indeed, apply a well-known result from measure theory (see Lemma 8) and the fact that $\left\{\xi<\omega^{+}: \tilde{\lambda}\left(V_{\xi}\right)>\delta\right\}$ is infinite for some (in fact, every) $0<\delta<1$.

So, if $K=\left\{\mathbf{1}_{A}: A \in \mathcal{A}\right\} \subset \Sigma\left(\{0,1\}^{\omega^{+}}\right) \subset \ell_{\infty}^{c}\left(\omega^{+}\right)$, then $K$ is a Corson compact space with respect to the $\mathrm{w}^{*}$-topology $\sigma\left(\ell_{\infty}\left(\omega^{+}\right), \ell_{1}\left(\omega^{+}\right)\right)$. Define the continuous map $T: \Omega \rightarrow K$ so that, for every $x \in \Omega, T(x)=\mathbf{1}_{A_{x}}$, where $A_{x}=\left\{\xi \in \omega^{+}: x \in V_{\xi}\right\}$. Observe that $A_{x} \in \mathcal{A}$ and, so, $T(x) \in K, \forall x \in \Omega$.

Let $L=T(\Omega) \subset K$. Then $L$ is a Corson compact space without property $(M)$, because $L$ is nonseparable but $L$ is the support of $\mu$, where $\mu=T(\tilde{\lambda})$ is the probability on $K$ image of $\tilde{\lambda}$ under $T$. So, as $L \subset K$, $K$ is also a Corson compact space without property $(M)$.

Let $I$ be the space $\omega^{+}$, with the discrete topology, and $X=\ell_{\infty}^{c}(I)$. Then, the dual space $X^{*}$ is

$$
X^{*}=\ell_{1}(I) \oplus_{1} M_{R}(c I \backslash I),
$$

where $M_{R}(c I \backslash I)$ is the space of Radon Borel measures $\nu$ on $\beta I$ such that $\operatorname{supp}(\nu) \subset c I \backslash I$ and $\oplus_{1}$ means $\ell_{1}$-sum (that is, if a Banach space $Y$ has the decomposition $Y=Y_{1} \oplus_{1} Y_{2}$ and $y \in Y$, with $y=y_{1}+y_{2}$ and $y_{1} \in Y_{1}, y_{2} \in Y_{2}$, then $\left.\|y\|=\left\|y_{1}\right\|+\left\|y_{2}\right\|\right)$. Observe that $\ell_{1}(I) \oplus_{1} M_{R}(c I \backslash I)$ can be considered as a 1-complemented closed subspace of $\left(\ell_{\infty}(I)\right)^{*}=\ell_{1}(I) \oplus_{1} M_{R}(\beta I \backslash I)$. 
The bidual of $X$ is

$$
X^{* *}=\ell_{\infty}(I) \oplus_{\infty} M_{R}(c I \backslash I)^{*},
$$

where $\oplus_{\infty}$ means $\ell_{\infty}$-sum (that is, if a Banach space $Y$ has the decomposition $Y=Y_{1} \oplus_{\infty} Y_{2}$ and $y \in Y$, with $y=y_{1}+y_{2}$ and $y_{1} \in Y_{1}, y_{2} \in Y_{2}$, then $\left.\|y\|=\sup \left\{\left\|y_{1}\right\|,\left\|y_{2}\right\|\right\}\right)$. Let $\pi_{1}, \pi_{2}: X^{* *} \rightarrow X^{* *}$ be the canonical projections onto $\ell_{\infty}(I)$ and $M_{R}(c I \backslash I)^{*}$, respectively. The subspaces $\pi_{1}\left(X^{* *}\right)=\ell_{\infty}(I)$ and $\pi_{2}\left(X^{* *}\right)=M_{R}(c I \backslash I)^{*}$ are $\mathrm{w}^{*}$-closed subspaces of $X^{* *}$. Moreover, the $\mathrm{w}^{*}$ topology $\sigma\left(X^{* *}, X^{*}\right)$ coincides on $\pi_{1}\left(X^{* *}\right)=\ell_{\infty}(I)$ with the $\sigma\left(\ell_{\infty}(I), \ell_{1}(I)\right)$ topology. For $x \in X^{* *}$ we write $x=\left(x_{1}, x_{2}\right)$, with $\pi_{1}(x)=x_{1} \in \ell_{\infty}(I)$ and $\pi_{2}(x)=x_{2} \in M_{R}(c I \backslash I)^{*}$. So, if $J: X \rightarrow X^{* *}$ is the canonical embedding and $f \in X$, then $J(f)=\left(f_{1}, f_{2}\right)$, where $f_{1}=\pi_{1}(f)=f$ and $\pi_{2}(f)=f_{2}$ is such that $f_{2}(\nu)=\nu(\check{f})=\int_{c I \backslash I} \check{f} d \nu$, for every $\nu \in M_{R}(c I \backslash I)$.

The map $\phi: \ell_{\infty}(I) \rightarrow X^{* *}$ such that $\phi(f)=(f, 0), \forall f \in \ell_{\infty}(I)$, is an isomorphism between $\ell_{\infty}(I)$ and $\pi_{1}\left(X^{* *}\right)$, for the norm-topologies and also for the $\sigma\left(\ell_{\infty}(I), \ell_{1}(I)\right)$-topology of $\ell_{\infty}(I)$ and the $\mathrm{w}^{*}$-topology of $\pi_{1}\left(X^{* *}\right)$. So, $H:=\phi(K)=\{(k, 0): k \in K\} \subset B\left(X^{* *}\right)$ is a Corson compact space without property $(M)$, which is homeomorphic to $K$. Since the family $\mathcal{A}$ is adequate (in particular, $B \in \mathcal{A}$ if $B \subset A$ and $A \in \mathcal{A}$ ), the subset $\left\{\mathbf{1}_{A}: A \in\right.$ $\mathcal{A}, A$ finite $\}$ of $K$ is dense in $K$. So, as $J\left(\mathbf{1}_{A}\right)=\left(\mathbf{1}_{A}, 0\right)$ when $A \subset \omega^{+}$is finite, we get that $H \cap J(X)$ is $\mathrm{w}^{*}$-dense in $H$, because

$$
\begin{aligned}
\phi\left(\left\{\mathbf{1}_{A}: A \in \mathcal{A}, A \text { finite }\right\}\right) & =\left\{\left(\mathbf{1}_{A}, 0\right): A \in \mathcal{A}, A \text { finite }\right\}= \\
& =J\left(\left\{\mathbf{1}_{A}: A \in \mathcal{A}, A \text { finite }\right\}\right) \subset H \cap J(X) .
\end{aligned}
$$

Claim 1. $d(H, J(X))=\frac{1}{2}$.

Indeed, pick $f \in K$ and assume that $f=\mathbf{1}_{A}$, for some $A \in \mathcal{A}$. If $|A|<\aleph_{0}$, clearly $\phi(f)=(f, 0)=J(f)$, that is, $\phi(f) \in J(X)$. Suppose that $|A|=\aleph_{0}$. Then $d(\phi(f), J(X))=\frac{1}{2}$ because:

(a) Clearly, $\left\|\phi(f)-\frac{1}{2} J(f)\right\|=\frac{1}{2}$, whence $d(\phi(f), J(X)) \leq \frac{1}{2}$.

(b) On the other hand, $\|\phi(f)-J(g)\| \geq \frac{1}{2}$ for every $g \in X$. Indeed, let $g \in X$ and assume that $\|\phi(f)-J(g)\| \leq \frac{1}{2}$. Then $\|f-g\| \leq \frac{1}{2}$ in $\ell_{\infty}(I)$, which implies that $\frac{1}{2} \leq g$ on $A$ (because $f=\mathbf{1}_{A}$ ) and so $\check{g} \geq \frac{1}{2}$ on $\bar{A}^{\beta I}$. Since $|A|=\aleph_{0}$, we can pick $p \in \bar{A}^{\beta I} \backslash I \subset c I \backslash I$. Let $\delta_{p} \in M_{R}(c I \backslash I)$ be such that $\delta_{p}(h)=\check{h}(p)$ for every $h \in \ell_{\infty}(I)$. Notice that $\left\|\delta_{p}\right\|=1$. Then, if $J(g)=\left(g, g_{2}\right)$, we have

$$
\left|(\phi(f)-J(g))\left(\delta_{p}\right)\right|=\left|-g_{2}\left(\delta_{p}\right)\right|=\left|-\int_{c I \backslash I} \check{g} \cdot d\left(\delta_{p}\right)\right|=|-\check{g}(p)| \geq \frac{1}{2} .
$$

Finally, recall that there are elements $A \in \mathcal{A}$ with $|A|=\aleph_{0}$. 
Claim 2. $d\left(\overline{\mathrm{CO}}^{w^{*}}(H), J(X)\right)=1$.

Indeed, first $d\left(\overline{\mathrm{cO}} w^{*}(H), J(X)\right) \leq 1$ because $\overline{\mathrm{co}} w^{*}(H) \subset B\left(X^{* *}\right)$. On the other hand, let $\nu:=\phi(\mu)$ be the probability on $\phi(L)$ image of $\mu$ under $\phi$. Since $\phi(L) \subset B\left(\pi_{1}\left(X^{* *}\right)\right)$ and $\pi_{1}\left(X^{* *}\right)$ is a convex $\mathrm{w}^{*}$-closed subset of $X^{* *}$, we conclude that $\overline{\mathrm{co}} w^{*}(\phi(L)) \subset B\left(\pi_{1}\left(X^{* *}\right)\right)$. So, as $r(\nu) \in \overline{\mathrm{Co}}^{w^{*}}(\phi(L))$, we get that $r(\nu)=\left(z_{0}, 0\right)$ for some $z_{0} \in B\left(\ell_{\infty}(I)\right)$. If $\xi \in I$, define $\pi_{\xi}: X^{* *} \rightarrow \mathbb{R}$ by $\pi_{\xi}\left(f_{1}, f_{2}\right)=f_{1}(\xi)$, for all $\left(f_{1}, f_{2}\right) \in X^{* *}=\ell_{\infty}(I) \oplus_{\infty} M_{R}(c I \backslash I)^{*}$. Observe that $\pi_{\xi}$ is a $\mathrm{w}^{*}$-continuous linear map on $X^{* *}$. So

$$
z_{0}(\xi)=\pi_{\xi}\left(z_{0}, 0\right)=\pi_{\xi}(r(\nu))=\int_{\phi(L)} \pi_{\xi}(k) d \nu=\int_{L} k(\xi) d \mu=\tilde{\lambda}\left(V_{\xi}\right) .
$$

Thus, for every $0<t<1$ we have, by construction, $\left|\left\{\xi \in I: z_{0}(\xi)>t\right\}\right|=$ $\left|\left\{\xi \in I: \tilde{\lambda}\left(V_{\xi}\right)>t\right\}\right|=\aleph_{1}$, and this implies that $\left\|z_{0}-g\right\| \geq 1$ in $\ell_{\infty}(I)$, for every $g \in X=\ell_{\infty}^{c}(I)$, whence $\left\|\left(z_{0}, 0\right)-J(g)\right\| \geq 1$ for every $g \in X$, that is, $d\left(\left(z_{0}, 0\right), J(X)\right) \geq 1$. Finally, we obtain $d\left(\overline{\mathrm{co}^{w}}(H), J(X)\right) \geq 1$ because $\left(z_{0}, 0\right) \in \overline{\mathrm{CO}}^{w^{*}}(\phi(L)) \subset \overline{\mathrm{CO}}^{w^{*}}(H)$.

And this completes the proof.

Remark. Theorem 1 gives, under $C H$, a negative answer to the following question posed in Problem 3 of [7]: if $X$ is a Banach space and $H \subset X$ a $\epsilon$-WRK, is $\operatorname{co}(H)$ a $\epsilon$-WRK?

We need the following well known result from measure theory.

Lemma 8 Let $(\Omega, \Sigma, \mu)$ be a measure space with $\mu$ positive and finite and $\left\{A_{n}\right\}_{n<\omega} \subset \Sigma$ be a sequence of measurable sets with $\mu\left(A_{n}\right)>\delta>0$ for all $n<\omega$ and some $\delta>0$. Then there exists an infinite subset $I \subset \omega$ such that $\cap_{n \in I} A_{n} \neq \emptyset$.

Proof. Consider the sequence $B_{n}=\cup_{k \geq n} A_{k}, n \geq 1$. The sequence $\left\{B_{n}\right\}_{n \geq 1}$ is decreasing and $\mu\left(B_{n}\right)>\delta$ for every $n \geq 1$. Hence $\mu\left(\cap_{n<\omega} B_{n}\right) \geq \delta$ and therefore $\cap_{n<\omega} B_{n} \neq \emptyset$. Choose $w \in \cap_{n<\omega} B_{n}$ and inductively a sequence $\left\{A_{n_{k}}\right\}_{k<\omega}, n_{k}<n_{k+1}$, such that $w \in A_{n_{k}}$ for all $k<\omega$. Then $I=\left\{n_{k}: k<\right.$ $\omega\}$ is the desired infinite subset.

Proposition 9 Let $I$ be an infinite set and $X=\left(c_{0}(I),\|\cdot\|_{\infty}\right)$. Then every $w^{*}$-compact subset $K \subset X^{* *}$ satisfies $d(K, X)=d\left(\overline{c o}^{w^{*}}(K), X\right)$.

Proof. First, recall that if $f \in X^{* *}=\ell_{\infty}(I)$, then

$$
d(f, X)=\sup \{|\check{f}(p)|: p \in \beta I \backslash I\} .
$$


Suppose that there exists a $\mathrm{w}^{*}$-compact subset $K \subset B\left(X^{* *}\right)$ such that $d(K, X)<d\left(\overline{\mathrm{co}}^{w^{*}}(K), X\right)$. Then we can find two real numbers $a, b$ such that

$$
d(K, X)<a<b<d\left(\overline{\mathrm{co}}^{w^{*}}(K), X\right) \leq 1 .
$$

Pick $z_{0} \in \overline{\mathrm{co}}^{w^{*}}(K)$ such that $d\left(z_{0}, X\right)>b$. So, there exist $\epsilon>0$ and $p_{0} \epsilon$ $\beta I \backslash I$ such that $\left|\check{z}_{0}\left(p_{0}\right)\right|>b+\epsilon$, for example, $\check{z}_{0}\left(p_{0}\right)>b+\epsilon$. Let $U \subset I$ be such that $p_{0} \in \bar{U}^{\beta I}$ and $z_{0}(j)>b+\epsilon, \forall j \in U$. Let $\mu$ be a Radon Borel probability on $K$ such that $z_{0}=r(\mu)$ and denote $A_{j}:=\{k \in K: k(j) \geq b\}, j \in U$, which is a closed subset of $K$.

Claim. $\mu\left(A_{j}\right)>\frac{\epsilon}{1-b}, \forall j \in U$.

Indeed, let $\pi_{j}: \ell_{\infty}(I) \rightarrow \mathbb{R}, j \in I$, be such that $\pi_{j}(f)=f(j)$ for every $f \in \ell_{\infty}(I)$. Observe that $\pi_{j}$ is a $\mathrm{w}^{*}$-continuous linear map on $\ell_{\infty}(I)$, for every $j \in I$. Thus, for every $j \in U$ we have

$$
\begin{aligned}
z_{0}(j) & =\pi_{j}\left(z_{0}\right)=\pi_{j}(r(\mu))=\int_{K} \pi_{j}(k) d \mu=\int_{K} k(j) d \mu= \\
& =\int_{A_{j}} k(j) d \mu+\int_{K \backslash A_{j}} k(j) d \mu \leq \mu\left(A_{j}\right)+\left(1-\mu\left(A_{j}\right)\right) b,
\end{aligned}
$$

and this implies

$$
\mu\left(A_{j}\right) \geq \frac{z_{0}(j)-b}{1-b}>\frac{\epsilon}{1-b} .
$$

Let $V_{0} \subset U$ be an arbitrary infinite subset. By Lemma 8 there exists an infinite countable subset $N_{0} \subset V_{0}$ such that $\emptyset \neq \cap_{j \in N_{0}} A_{j} \subset K$. Pick $x_{0} \in \cap_{j \in N_{0}} A_{j}$. Then for every $q \in{\overline{N_{0}}}^{\beta I} \backslash I$ we have $\check{x}_{0}(q) \geq b$, which implies $d\left(x_{0}, X\right) \geq b$, a contradiction, because $x_{0}$ belongs to $K$.

If $(X, \tau)$ is a topological space, a subset $K \subset X$ is said to be regular in $X$ if and only if the interior set $\operatorname{int}(K)$ is dense in $K$.

Corollary 10 Let $I$ be an infinite set, $H \subset \beta I \backslash I$ a compact subset which is regular in $\beta I \backslash I$, and $Y_{H}=\left\{f \in \ell_{\infty}(I): \breve{f}_{\mid H}=0\right\}$. Then for every $w^{*}$-compact subset $K \subset \ell_{\infty}(I)$ we have $d\left(K, Y_{H}\right)=d\left(\overline{c o}^{w^{*}}(K), Y_{H}\right)$.

Proof. First, observe that $d\left(z, Y_{H}\right)=\sup \{|\check{z}(x)|: x \in H\}$ for every $z \in$ $\ell_{\infty}(I)$. Suppose that there exist a ${ }^{*}$-compact subset $K \subset B\left(\ell_{\infty}(I)\right)$ and real numbers $a, b$ such that:

$$
d\left(K, Y_{H}\right)<a<b<d\left(\overline{\mathrm{co}}^{w^{*}}(K), Y_{H}\right) \leq 1 .
$$

Let $z_{0} \in \overline{\mathrm{co}}^{w^{*}}(K)$ be such that $d\left(z_{0}, Y_{H}\right)>b$. Since $\operatorname{int}(H)$ is dense in $H$, there exists $p_{0} \in \operatorname{int}(H)$ such that, for example, $\check{z}_{0}\left(p_{0}\right)>b+\epsilon$, for some 
$\epsilon>0$. Let $U \subset I$ be an infinite subset such that $p_{0} \in \bar{U}^{\beta I} \backslash I \subset H$ and $z_{0}(j)>b+\epsilon, \forall j \in U$. By an argument similar to that of Proposition 9, we find an infinite countable subset $N_{0} \subset U$ and a vector $x_{0} \in K$ such that $\check{x}_{0}(q) \geq b$, for every $q \in{\overline{N_{0}}}^{\beta I} \backslash I \subset H$, which implies $d\left(x_{0}, Y_{H}\right) \geq b$, a contradiction, because $x_{0} \in K$ and $d\left(K, Y_{H}\right) \leq a<b$.

We now prove Theorem 3 and Corollary 4.

Proof of Theorem 3. Suppose that there exist a $\mathrm{w}^{*}$-compact subset $K \subset$ $B\left(X^{* *}\right)$ and real numbers $a, b$ such that:

$$
d(K, X)<a<b<d\left(\overline{\mathrm{CO}}^{w^{*}}(K), X\right) .
$$

Pick $z_{0} \in \overline{\mathrm{co}}^{w^{*}}(K)$ with $d\left(z_{0}, X\right)>b$. Since $X \in J$ we can choose a sequence $\left\{x_{n}^{*}\right\}_{n \geq 1} \subset \mathfrak{S}\left(B\left(X^{*}\right), z_{0}, b\right)$ such that $x_{n}^{*} \stackrel{w^{*}}{\rightarrow} 0$. Let $T: X \rightarrow c_{0}:=c_{0}(\mathbb{N})$ be such that $T(x)=\left(x_{n}^{*}(x)\right)_{n \geq 1}, \forall x \in X$. Clearly, $T$ is a linear continuous map with $\|T\| \leq 1$. Let $L=T^{* *}(K)$, which is a $\mathrm{w}^{*}$-compact subset of $B\left(\ell_{\infty}\right)$.

Claim 1. $d\left(L, c_{0}\right) \leq d(K, X)$.

Indeed, let $c_{0}^{\perp}=\left\{f \in c_{0}^{* * *}:\langle f, u\rangle=0, \forall u \in c_{0}\right\}$ and pick $v \in B\left(c_{0}^{\perp}\right)$. Then $\left\|T^{* * *}(v)\right\| \leq 1$ and for every $x \in X$ we have:

$$
\left\langle T^{* * *}(v), x\right\rangle=\left\langle v, T^{* *} x\right\rangle=\langle v, T x\rangle=0 .
$$

So, $T^{* * *}\left(B\left(c_{0}^{\perp}\right)\right) \subset B\left(X^{\perp}\right)$. Hence, if $k \in K$ and $T^{* *}(k)=: h \in L$ we have:

$$
\begin{aligned}
d\left(h, c_{0}\right) & =\sup \left\{\langle v, h\rangle: v \in B\left(c_{0}^{\perp}\right)\right\}= \\
& =\sup \left\{\left\langle v, T^{* *}(k)\right\rangle: v \in B\left(c_{0}^{\perp}\right)\right\}=\sup \left\{\left\langle T^{* * *}(v), k\right\rangle: v \in B\left(c_{0}^{\perp}\right)\right\} \leq \\
& \leq \sup \left\{\langle w, k\rangle: w \in B\left(X^{\perp}\right)\right\}=d(k, X) .
\end{aligned}
$$

Claim 2. If $w_{0}:=T^{* *}\left(z_{0}\right) \in \overline{\mathrm{CO}} w^{*}(L)$, then $d\left(w_{0}, c_{0}\right) \geq b$.

Indeed, let $\left\{e_{n}\right\}_{n \geq 1}$ be the canonical basis of $\ell_{1}$, which satisfies $T^{*}\left(e_{n}\right)=$ $x_{n}^{*}, \forall n \geq 1$. Since $x_{n}^{*} \in \mathfrak{S}\left(B\left(X^{*}\right), z_{0}, b\right)$, then

$$
\left\langle w_{0}, e_{n}\right\rangle=\left\langle T^{* *}\left(z_{0}\right), e_{n}\right\rangle=\left\langle z_{0}, T^{*}\left(e_{n}\right)\right\rangle=\left\langle z_{0}, x_{n}^{*}\right\rangle \geq b .
$$

Let $\psi$ be a $\mathrm{w}^{*}$-limit point of $\left\{e_{n}\right\}_{n \geq 1}$ in $\left(\ell_{\infty}^{*}, w^{*}\right)$. Clearly, $\psi \in B\left(c_{0}^{\perp}\right)$ and also $\psi\left(w_{0}\right) \geq b$ by $(2.1)$. So, $d\left(w_{0}, c_{0}\right) \geq b$.

Therefore, $L \subset B\left(\ell_{\infty}\right)$ is a $\mathrm{w}^{*}$-compact subset such that

$$
d\left(L, c_{0}\right) \leq d(K, X)<a<b \leq d\left(w_{0}, c_{0}\right) \leq d\left(\overline{\mathrm{co}}^{*}(L), c_{0}\right),
$$

a contradiction to Proposition 9. 
Of course, not every Banach space has property $J$. Indeed, if $X$ is a non-reflexive Grothendieck Banach space (for example, if $X=\ell_{\infty}(I)$ with $I$ infinite), then clearly $X$ does not have property $J$. Moreover, $X$ cannot be isomorphically embedded into a Banach space with property $J$.

However, the family of Banach spaces fulfilling property $J$ is very large. For example, this family includes the class of Banach spaces $X$ whose dual unit ball $\left(B\left(X^{*}\right), \mathrm{w}^{*}\right)$ is angelic in the $\mathrm{w}^{*}$-topology. Recall that every WCG (even every WLD) Banach space belongs to this class (see [2]).

Proof of Corollary 4. The proof of this fact is standard and well known. Let us prove that if $z_{0} \in B\left(X^{* *}\right) \backslash X$ and $0<b<d\left(z_{0}, X\right)$, then

$$
0 \in{\overline{\mathfrak{S}\left(B\left(X^{*}\right), z_{0}, b\right)}}^{\sigma\left(X^{*}, X\right)} .
$$

Find $\psi \in S\left(X^{\perp}\right) \subset X^{* * *}$ such that $\psi\left(z_{0}\right)>b$. As $B\left(X^{*}\right)$ is $\mathrm{w}^{*}$-dense in $B\left(X^{* * *}\right)$ and $\psi\left(z_{0}\right)>b$, then

$$
\psi \in \overline{\mathfrak{S}\left(B\left(X^{*}\right), z_{0}, b\right)}{ }^{\sigma\left(X^{* * *}, X^{* *}\right)},
$$

whence we obtain

$$
0 \in{\overline{\mathfrak{S}\left(B\left(X^{*}\right), z_{0}, b\right)}}^{\sigma\left(X^{*}, X\right)},
$$

because $\psi \in X^{\perp}$. Finally, it is enough to apply the fact that $\left(B\left(X^{*}\right), \mathrm{w}^{*}\right)$ is angelic.

Now we prove some auxiliary facts. If $X$ is a Banach space, let $I_{X}: X \rightarrow X$ denote the identity map of $X, J_{X}: X \rightarrow X^{* *}$ the canonical embedding of $X$ into $X^{* *}$ and $R_{X}: X^{* * *} \rightarrow X^{*}$ the canonical restriction map such that $\left\langle R_{X}(z), x\right\rangle=\left\langle z, J_{X}(x)\right\rangle$, for every $z \in X^{* * *}$ and every $x \in X$. Notice that $R_{X}=\left(J_{X}\right)^{*}$ and that $R_{X} \circ J_{X^{*}}=I_{X^{*}}$.

It is well-known that $J_{X^{*}}\left(X^{*}\right)$ is 1-complemented into $X^{* * *}$, by means of the projection $P_{X}: X^{* * *} \rightarrow X^{* * *}$ such that $P_{X}=J_{X^{*}} \circ R_{X}$. Since $\operatorname{ker}\left(P_{X}\right)=$ $\left\{z \in X^{* * *}:\left\langle z, J_{X}(x)\right\rangle=0, \forall x \in X\right\}=X^{\perp}$, we have the decomposition $X^{* * *}=X^{\perp} \oplus J_{X^{*}}\left(X^{*}\right)$. The subspace $X^{\perp}$ is complemented in $X^{* * *}$ by means of the projection $Q_{X}: X^{* * *} \rightarrow X^{* * *}$ such that $Q_{X}=I_{X^{* * *}}-P_{X}$. Observe that $1 \leq\left\|Q_{X}\right\| \leq 2$ and that:

$$
B\left(X^{\perp}\right) \subset Q_{X}\left(B\left(X^{* * *}\right)\right) \subset\left\|Q_{X}\right\| \cdot B\left(X^{\perp}\right) \subset 2 B\left(X^{\perp}\right) .
$$

Lemma 11 Let $X$ be a Banach space and $Q_{X}: X^{* * *} \rightarrow X^{* * *}$ be the canonical projection onto $X^{\perp}$. Assume that $Y \subset X$ is a closed subspace. Then, for every $u \in Y^{* *}$ (considered $Y^{* *}$ as a subspace of $X^{* *}$ ) we have:

$$
d(u, X) \leq d(u, Y) \leq\left\|Q_{X}\right\| \cdot d(u, X) \leq 2 d(u, X) .
$$


Proof. First, it is clear that $d(u, X) \leq d(u, Y)$, because $Y \subset X$.

In the following we distinguish $X$ from $J_{X}(X), Y$ from $J_{Y}(Y)$, etc. Let $i: Y \rightarrow X$ denote the inclusion map. Then $i^{*}: X^{*} \rightarrow Y^{*}$ is a quotient map, $i^{* *}: Y^{* *} \rightarrow X^{* *}$ is an inclusion map such that $\left(i^{* *}\right)_{\mid Y}=i$, and $i^{* * *}$ : $X^{* * *} \rightarrow Y^{* * *}$ is a quotient map such that $\left(i^{* * *}\right)_{\mid X^{*}}=i^{*}$. Observe that $i^{* * *}\left(B\left(X^{* * *}\right)\right)=B\left(Y^{* * *}\right)$. It is easy to see that $J_{X} \circ i=i^{* *} \circ J_{Y}$ and that $J_{Y^{*}} \circ i^{*}=i^{* * *} \circ J_{X^{*}}$, whence we obtain

$$
i^{*} \circ R_{X}=i^{*} \circ\left(J_{X}\right)^{*}=\left(J_{X} \circ i\right)^{*}=\left(i^{* *} \circ J_{Y}\right)^{*}=\left(J_{Y}\right)^{*} \circ i^{* * *}=R_{Y} \circ i^{* * *} .
$$

Claim. $Q_{Y} \circ i^{* * *}=i^{* * *} \circ Q_{X}$.

Indeed, we have

$$
\begin{aligned}
Q_{Y} \circ i^{* * *} & =\left(I_{Y^{* * *}}-J_{Y^{*}} \circ R_{Y}\right) \circ i^{* * *}=i^{* * *}-J_{Y^{*}} \circ R_{Y} \circ i^{* * *}= \\
& =i^{* * *}-J_{Y^{*}} \circ i^{*} \circ R_{X}=i^{* * *}-i^{* * *} \circ J_{X^{*}} \circ R_{X}= \\
& =i^{* * *} \circ\left(I_{X^{* * *}}-J_{X^{*}} \circ R_{X}\right)=i^{* * *} \circ Q_{X} .
\end{aligned}
$$

From the Claim we obtain $\left\|Q_{Y}\right\| \leq\left\|Q_{X}\right\|$ and

$$
\begin{aligned}
B\left(Y^{\perp}\right) \subset Q_{Y}\left(B\left(Y^{* * *}\right)\right) & =Q_{Y}\left(i^{* * *}\left(B\left(X^{* * *}\right)\right)\right)= \\
& =i^{* * *}\left(Q_{X}\left(B\left(X^{* * *}\right)\right)\right) \subset i^{* * *}\left(\left\|Q_{X}\right\| \cdot B\left(X^{\perp}\right)\right) .
\end{aligned}
$$

Thus, if $u \in Y^{* *}$, we finally get

$$
\begin{aligned}
d\left(u, J_{Y}(Y)\right) & =\sup \left\{\langle z, u\rangle: z \in B\left(Y^{\perp}\right)\right\} \\
& \leq \sup \left\{\left\langle i^{* * *}(w), u\right\rangle: w \in\left\|Q_{X}\right\| \cdot B\left(X^{\perp}\right)\right\} \\
& =\left\|Q_{X}\right\| \cdot \sup \left\{\left\langle w, i^{* *}(u)\right\rangle: w \in B\left(X^{\perp}\right)\right\} \\
& =\left\|Q_{X}\right\| \cdot d\left(i^{* *}(u), J_{X}(X)\right) \\
& \leq 2 d\left(i^{* *}(u), J_{X}(X)\right) .
\end{aligned}
$$

Let us prove our extension of the Krein-Šmulian Theorem.

Proof of Theorem 5. Suppose that there exist a closed subspace $Z \subset X$ and a $\mathrm{w}^{*}$-compact subset $K \subset B\left(X^{* *}\right)$ such that

$$
d\left(\overline{\mathrm{co}}^{w^{*}}(K), Z\right)>5 d(K, Z) .
$$

Then we can find $z_{0} \in \overline{\mathrm{co}}^{w^{*}}(K)$ and $a, b>0$ such that

$$
d\left(z_{0}, Z\right)>b>5 a>5 d(K, Z) .
$$

Pick $\psi \in S\left(Z^{\perp}\left(X^{* * *}\right)\right)$ with $\psi\left(z_{0}\right)>b$. 
Step 1. Since $\psi\left(z_{0}\right)>b$, there exists $x_{1}^{*} \in S\left(X^{*}\right)$ such that $x_{1}^{*}\left(z_{0}\right)>b$. So, as $z_{0} \in \overline{\mathrm{co}}^{w^{*}}(K)$ we can find $\eta_{1} \in \operatorname{co}(K)$ with

$$
\eta_{1}=\sum_{i=1}^{n_{1}} \lambda_{1 i} \eta_{1 i}, \quad \eta_{1 i} \in K, \quad \lambda_{1 i} \geq 0, \sum_{i=1}^{n_{1}} \lambda_{1 i}=1,
$$

such that $x_{1}^{*}\left(\eta_{1}\right)>b$. Since $d\left(\eta_{1 i}, Z\right)<a$ we have the decomposition $\eta_{1 i}=$ $\eta_{1 i}^{1}+\eta_{1 i}^{2}$ with $\eta_{1 i}^{1} \in Z$ and $\eta_{1 i}^{2} \in a B\left(X^{* *}\right)$.

Step 2. Let $Y_{1}=\left[\left\{\eta_{1 i}^{1}: 1 \leq i \leq n_{1}\right\}\right] \subset Z$. Since $\operatorname{dim}\left(Y_{1}\right) \leq n_{1}<\infty$, $\psi\left(z_{0}\right)>b$ and $\psi \in Y_{1}^{\perp}\left(X^{* * *}\right)$, there exists $x_{2}^{*} \in S\left(X^{*}\right)$ such that $x_{2}^{*}\left(z_{0}\right)>b$ and $x_{2 \nmid Y_{1}}^{*}=0$. So, as $x_{i}^{*}\left(z_{0}\right)>b, i=1,2$, and $z_{0} \in \overline{\mathrm{CO}}^{w^{*}}(K)$, we can find $\eta_{2} \in \operatorname{co}(K)$ with

$$
\eta_{2}=\sum_{i=1}^{n_{2}} \lambda_{2 i} \eta_{2 i}, \quad \eta_{2 i} \in K, \quad \lambda_{2 i} \geq 0, \sum_{i=1}^{n_{2}} \lambda_{2 i}=1,
$$

such that $x_{i}^{*}\left(\eta_{2}\right)>b, i=1,2$. Since $d\left(\eta_{2 i}, Z\right)<a$ we have the decomposition $\eta_{2 i}=\eta_{2 i}^{1}+\eta_{2 i}^{2}$ with $\eta_{2 i}^{1} \in Z$ and $\eta_{2 i}^{2} \in a B\left(X^{* *}\right)$.

By reiteration, we obtain the sequences $\left\{x_{n}^{*}\right\}_{n \geq 1} \subset S\left(X^{*}\right), \eta_{k} \in \operatorname{co}(K)$ with

$$
\begin{gathered}
\eta_{k}=\sum_{i=1}^{n_{k}} \lambda_{k i} \eta_{k i}, \eta_{k i} \in K, \quad \lambda_{k i} \geq 0, \sum_{i=1}^{n_{k}} \lambda_{k i}=1, \\
\eta_{k i}=\eta_{k i}^{1}+\eta_{k i}^{2} \text { with } \eta_{k i}^{1} \in Z \text { and } \eta_{k i}^{2} \in a B\left(X^{* *}\right), \quad k \geq 1,
\end{gathered}
$$

such that $x_{i}^{*}\left(\eta_{k}\right)>b, i=1, \ldots, k$, and $x_{k+1 \mid Y_{k}}^{*}=0$, where

$$
Y_{k}=\left[\left\{\eta_{i j_{i}}^{1}: i=1, \ldots, k ; 1 \leq j_{i} \leq n_{i}\right\}\right] \subset Y_{k+1} \subset Z .
$$

Let $Y=\overline{\cup_{k \geq 1} Y_{k}} \subset Z$ and $K_{1}=\left(K+a B\left(X^{* *}\right)\right) \cap Y^{* *}$. Then $Y$ is a closed separable subspace of $Z$ and $K_{1}$ is a $\mathrm{w}^{*}$-compact subset of $Y^{* *}$ (considered $Y^{* *}$ canonically embedded into $\left.Z^{* *} \subset X^{* *}\right)$. Observe that $\left\{\eta_{i j_{i}}^{1}: i \geq 1,1 \leq\right.$ $\left.j_{i} \leq n_{i}\right\} \subset K_{1}$. By Lemma 11 , since $K_{1} \subset Y^{* *}$ and $d\left(K_{1}, Z\right) \leq 2 a$, we have $d\left(K_{1}, Y\right) \leq 4 a$ (in fact, $d\left(K_{1}, Y\right) \leq 2\left\|Q_{Z}\right\| a \leq 2\left\|Q_{X}\right\| a \leq 4 a$ ). As $Y$ has property $J$ (because $Y$ is separable and, so, WCG, see Corollary 4), we get $d\left(\overline{\mathrm{CO}}^{w^{*}}\left(K_{1}\right), Y\right)=d\left(K_{1}, Y\right)$, whence $d\left(\overline{\mathrm{CO}}^{w^{*}}\left(K_{1}\right), Y\right) \leq 4 a$.

Let $\eta_{0}$ be a $\mathrm{w}^{*}$-limit point of $\left\{\eta_{k}\right\}_{k \geq 1}$ in $X^{* *}$.

Claim 1. $d\left(\eta_{0}, Y\right) \leq 5 a$.

Indeed, first

$$
\eta_{0} \in \overline{\mathrm{CO}^{w}} w^{*}\left(\left\{\eta_{i j_{i}}: i \geq 1,1 \leq j_{i} \leq n_{i}\right\}\right) \subset \overline{\mathrm{CO}} w^{*}\left(K_{1}\right)+a B\left(X^{* *}\right) .
$$

On the other hand, $d\left(\overline{\mathrm{co}}^{w^{*}}\left(K_{1}\right), Y\right) \leq 4 a$. Hence, $d\left(\eta_{0}, Y\right) \leq 5 a$. 
Claim 2. $d\left(\eta_{0}, Y\right) \geq b$.

Indeed, let $\phi \in B\left(X^{* *}\right)$ be a $\mathrm{w}^{*}$-limit point of $\left\{x_{n}^{*}\right\}_{n \geq 1}$. Since $x_{n}^{*}\left(\eta_{k}\right)>b$ if $k \geq n$, then $x_{n}^{*}\left(\eta_{0}\right) \geq b, \forall n \geq 1$, whence $\phi\left(\eta_{0}\right) \geq b$. Moreover, $\phi \in$ $Y^{\perp}\left(X^{* * *}\right)$ because $x_{n+1 \mid Y_{n}}^{*}=0$ and $Y_{n} \subset Y_{n+1}$. Hence, $d\left(\eta_{0}, Y\right) \geq \phi\left(\eta_{0}\right) \geq b$.

Since $b>5 a$ we get a contradiction and this completes the proof.

Proof of Theorem 6. Suppose that there exist a closed subspace $Z \subset X$ and a $\mathrm{w}^{*}$-compact subset $K \subset B\left(X^{* *}\right)$, with $Z \cap K \mathrm{w}^{*}$-dense in $K$, such that $d\left(\overline{\mathrm{CO}} w^{*}(K), Z\right)>2 d(K, Z)$. Then we can find $z_{0} \in \overline{\mathrm{CO}} w^{*}(K)$ and $a, b>0$ such that $d\left(z_{0}, Z\right)>b>2 a>2 d(K, Z)$. Pick $\psi \in S\left(Z^{\perp}\left(X^{* * *}\right)\right)$ such that $\psi\left(z_{0}\right)>b$. We follow the argument of Theorem 5 with the following changes:

(i) As $Z \cap K$ is $\mathrm{w}^{*}$-dense in $K$ we choose $\eta_{k} \in \operatorname{co}(Z \cap K)$ with $\eta_{k}=$ $\sum_{i=1}^{n_{k}} \lambda_{k i} \eta_{k i}, \eta_{k i} \in Z \cap K$ and $\lambda_{k i} \geq 0, \sum_{i=1}^{n_{k}} \lambda_{k i}=1$;

(ii) Define

$$
\begin{aligned}
Y_{k}= & {\left[\left\{\eta_{i j_{i}}: i=1, \ldots, k ; 1 \leq j_{i} \leq n_{i}\right\}\right], Y=\overline{\cup_{k \geq 1} Y_{k}} \subset Z \text { and } } \\
& K_{1}=\mathrm{w}^{*}-\operatorname{cl}\left(\left\{\eta_{i j_{i}}: i \geq 1,1 \leq j_{i} \leq n_{i}\right\}\right) \subset Y^{* *} \cap K .
\end{aligned}
$$

Clearly, $d\left(K_{1}, Z\right) \leq d(K, Z)<a$, whence $d\left(K_{1}, Y\right) \leq 2 d\left(K_{1}, Z\right) \leq 2 a$ (in fact, $\left.d\left(K_{1}, Y\right) \leq\left\|Q_{Z}\right\| a \leq\left\|Q_{X}\right\| a \leq 2 a\right)$. Since $Y$ is separable, we have $d\left(\overline{\mathrm{co}}^{w^{*}}\left(K_{1}\right), Y\right)=d\left(K_{1}, Y\right) \leq 2 a$. Finally, every $\mathrm{w}^{*}$-limit point $\eta_{0}$ of $\left\{\eta_{k}\right\}_{k \geq 1}$ in $X^{* *}$ satisfies $\eta_{0} \in \overline{\mathrm{CO}}^{w^{*}}\left(K_{1}\right), d\left(\eta_{0}, Y\right) \leq 2 a$ and $d\left(\eta_{0}, Y\right) \geq b$, a contradiction.

Remarks. (1) The argument of Theorem 5 in fact yields the following

$$
d\left(\overline{\mathrm{co}}^{w^{*}}(K), Z\right) \leq\left(2\left\|Q_{Z}\right\|+1\right) d(K, Z) \leq\left(2\left\|Q_{X}\right\|+1\right) d(K, Z) \leq 5 d(K, Z) .
$$

In Theorem 6 we also obtain

$$
d\left(\overline{\mathrm{co}}^{w^{*}}(K), Z\right) \leq\left\|Q_{Z}\right\| d(K, Z) \leq\left\|Q_{X}\right\| d(K, Z) \leq 2 d(K, Z) .
$$

(2) Let $Y \subset X$ be a subspace of the Banach space $X$ and assume that $d\left(\overline{\mathrm{co}}^{w^{*}}(K), X\right) \leq M d(K, X)$ for some $1 \leq M<\infty$ and every $\mathrm{w}^{*}$ compact subset $K \subset X^{* *}$. Then using the fact that $d(z, X) \leq d(z, Y) \leq$ $\left\|Q_{X}\right\| d(z, X) \leq 2 d(z, X)$, for every $z \in Y^{* *}$, it can be proved easily that $d\left(\overline{\mathrm{co}} w^{*}(K), Y\right) \leq M\left\|Q_{X}\right\| d(K, Y) \leq 2 M d(K, X)$, for every $\mathrm{w}^{*}$-compact subset $K \subset Y^{* *}$.

A subset $A \subset X^{*}$ is said to be fragmented by the norm of $X^{*}$ (see [6, p. 81], [10]) if for every subset $B \subset A$ and every $\epsilon>0$ there exists a w*open subset $V \subset X^{*}$ such that $V \cap B \neq \emptyset$ and $\operatorname{diam}(V \cap B) \leq \epsilon$, where $\operatorname{diam}(V \cap B)$ means the diameter of $V \cap B$. In order to prove Corollary 13 and Theorem 7 we need the following lemma. 
Lemma 12 Let $X$ be a Banach space, $Z \subset X^{*}$ a subspace and $K \subset B\left(X^{*}\right)$ $a w^{*}$-compact subset such that there exist $a, b>0$ with:

$$
d(K, Z)<a<b<d\left(\overline{c o}^{w^{*}}(K), Z\right) .
$$

Then there exist $z_{0} \in \overline{c o}^{w^{*}}(K)$ and $\psi \in S\left(Z^{\perp}\left(X^{* *}\right)\right)$ with $\psi\left(z_{0}\right)>b$ such that, if $\mu$ is a Radon Borel probability measure on $K$ with barycentre $r(\mu)=z_{0}$, then: (a) $\mu$ is atomless; (b) if $H=\operatorname{supp}(\mu)$, for every $w^{*}$-open subset $V$ of $X^{*}$ with $V \cap H \neq \emptyset$ there exists $\xi \in \overline{c o}^{w^{*}}(V \cap H)$ such that $\psi(\xi)>b$; and (c) $H$ is not fragmented by the norm of $X^{*}$.

Proof. Pick $z \in \overline{\mathrm{co}}^{w^{*}}(K)$ and $\psi \in S\left(Z^{\perp}\left(X^{* *}\right)\right)$ such that $\psi(z)>b+\epsilon$ for some $\epsilon>0$. By the Bishop-Phelps theorem, there exists $\phi \in S\left(X^{* *}\right)$ with $\|\psi-\phi\| \leq \epsilon / 4$ such that $\phi$ attains its maximum value on $\overline{\mathrm{co}}^{w^{*}}(K)$ in some $z_{0} \in \overline{\mathrm{co}} w^{*}(K)$. So:

$$
\begin{gathered}
\phi\left(z_{0}\right) \geq \phi(z)=\psi(z)+(\phi-\psi)(z)>b+\epsilon-\frac{1}{4} \epsilon=b+\frac{3}{4} \epsilon, \\
\psi\left(z_{0}\right)=\phi\left(z_{0}\right)+(\psi-\phi)\left(z_{0}\right)>b+\frac{3}{4} \epsilon-\frac{1}{4} \epsilon=b+\frac{1}{2} \epsilon \quad \text { and } \\
\forall k \in K, \phi(k)=\psi(k)+(\phi-\psi)(k)<a+\frac{1}{4} \epsilon<b+\frac{3}{4} \epsilon<\phi\left(z_{0}\right) .
\end{gathered}
$$

In particular, observe that $z_{0} \notin K$ by (2.4).

(a) Let $\mu$ be a Radon Borel probability on $K$ with barycentre $r(\mu)=z_{0}$ and suppose that $\mu$ has some atom, that is, there exist $0<\lambda \leq 1$ and $k_{0} \in K$ such that $\mu=\lambda \cdot \delta_{k_{0}}+\mu_{1}, \mu_{1} \geq 0$. If $\lambda=1$ then $\mu=\delta_{k_{0}}$, whence $r(\mu)=k_{0} \in K$, which is impossible because $r(\mu)=z_{0} \notin K$ by (2.4). So, $0<\lambda<1$, i.e., $\mu_{1} \neq 0$ and $\left\|\mu_{1}\right\|=1-\lambda>0$. Then $\mu=\lambda \cdot \delta_{k_{0}}+(1-\lambda) \frac{\mu_{1}}{\left\|\mu_{1}\right\|}$ and

$$
z_{0}=r(\mu)=\lambda k_{0}+(1-\lambda) r\left(\frac{\mu_{1}}{\left\|\mu_{1}\right\|}\right),
$$

whence, since $\phi\left(k_{0}\right)<\phi\left(z_{0}\right)$ (by $\left.(2.4)\right)$ and $\phi\left(r\left(\frac{\mu_{1}}{\left\|\mu_{1}\right\|}\right)\right) \leq \phi\left(z_{0}\right)$ (because $\left.r\left(\frac{\mu_{1}}{\left\|\mu_{1}\right\|}\right) \in \overline{\mathrm{CO}}^{w^{*}}(K)\right)$, we get

$$
\phi\left(z_{0}\right)=\lambda \phi\left(k_{0}\right)+(1-\lambda) \phi\left(r\left(\frac{\mu_{1}}{\left\|\mu_{1}\right\|}\right)\right)<\lambda \phi\left(z_{0}\right)+(1-\lambda) \phi\left(z_{0}\right)=\phi\left(z_{0}\right),
$$

a contradiction.

(b) Let $H=\operatorname{supp}(\mu)$ and suppose that there exists a $\mathrm{w}^{*}$-open subset $V$ of $X^{*}$ with $V \cap H \neq \emptyset$ such that $\psi(\xi) \leq b$, for every $\xi \in \overline{\mathrm{co}}^{w^{*}}(V \cap H)$. Let $\mu_{1}=$ $\mu_{\uparrow V \cap H}$ denote the restriction of $\mu$ to $V \cap H$ (that is, $\mu_{1}(B)=\mu(B \cap V \cap H)$, for every Borel subset $B \subset K)$ and $\mu_{2}:=\mu-\mu_{1}$. Observe that $\mu_{1}, \mu_{2}$ are 
positive measures such that $\mu_{1} \neq 0$ (because $\left.\emptyset \neq V \cap H=V \cap \operatorname{supp}(\mu)\right)$ and $\mu_{2} \neq 0$ (if $\mu_{2}=0$, i.e., $\mu=\mu_{1}=\mu_{\uparrow V \cap H}$, then $z_{0}=r(\mu) \in \overline{\mathrm{co}} w^{*}(V \cap H)$ ) and $\psi\left(z_{0}\right) \leq b$, a contradiction to (2.3)). Thus, we have the decomposition $\mu=\mu_{1}+\mu_{2}$ and so:

$$
z_{0}=r(\mu)=\left\|\mu_{1}\right\| \cdot r\left(\frac{\mu_{1}}{\left\|\mu_{1}\right\|}\right)+\left\|\mu_{2}\right\| \cdot r\left(\frac{\mu_{2}}{\left\|\mu_{2}\right\|}\right) .
$$

Since $r\left(\frac{\mu_{1}}{\left\|\mu_{1}\right\|}\right) \in \overline{\mathrm{co}} w^{*}(V \cap H)$, then $\psi\left(r\left(\frac{\mu_{1}}{\left\|\mu_{1}\right\|}\right)\right) \leq b$, whence $\phi\left(r\left(\frac{\mu_{1}}{\left\|\mu_{1}\right\|}\right)\right) \leq b+\frac{1}{4} \epsilon$ (because $\|\psi-\phi\| \leq \epsilon / 4)$. Therefore, taking into account that $r\left(\frac{\mu_{2}}{\left\|\mu_{2}\right\|}\right) \in$ $\overline{\mathrm{co}}^{w^{*}}(K)$ and $(2.2)$ we get

$$
\begin{aligned}
\phi\left(z_{0}\right) & =\left\|\mu_{1}\right\| \phi\left(r\left(\frac{\mu_{1}}{\left\|\mu_{1}\right\|}\right)\right)+\left\|\mu_{2}\right\| \phi\left(r\left(\frac{\mu_{2}}{\left\|\mu_{2}\right\|}\right)\right) \leq \\
& \leq\left\|\mu_{1}\right\|\left(b+\frac{1}{4} \epsilon\right)+\left\|\mu_{2}\right\| \phi\left(z_{0}\right)<\left\|\mu_{1}\right\| \phi\left(z_{0}\right)+\left\|\mu_{2}\right\| \phi\left(z_{0}\right)=\phi\left(z_{0}\right),
\end{aligned}
$$

a contradiction.

(c) Let $\eta=b-a$ and suppose that $H$ is fragmented by the norm of $X^{*}$. Then there exists a $\mathrm{w}^{*}$-open subset $V$ such that $V \cap H \neq \emptyset$ and $\operatorname{diam}(V \cap$ $H)<\frac{\eta}{2}$. Therefore, if $h_{0} \in V \cap H$, then $\overline{\mathrm{co}}^{w^{*}}(V \cap H) \subset B\left(h_{0} ; \eta / 2\right)(=$ closed ball with center $h_{0}$ and radius $\left.\eta / 2\right)$. Hence, for every $\xi \in \overline{\mathrm{CO}}^{w^{*}}(V \cap H)$ we have

$$
\psi(\xi) \leq \psi\left(h_{0}\right)+\frac{\eta}{2} \leq d\left(h_{0}, Z\right)+\frac{\eta}{2}<a+\frac{\eta}{2}<b,
$$

a contradiction to (b).

Corollary 13 Let $X$ be a Banach space, $Z \subset X^{*}$ a subspace and $K \subset$ $X^{*}$ a $w^{*}$-compact subset which is fragmented by the norm of $X^{*}$. Then $d\left(\overline{c o} w^{*}(K), Z\right)=d(K, Z)$.

Proof. This follows immediately from Lemma 12. It also follows from $[10$, Theorem 2.3] where it is proved that $\overline{\mathrm{co}}(K)=\overline{\mathrm{co}} w^{*}(K)$ whenever $K \subset X^{*}$ is $\mathrm{w}^{*}$-compact subset such that $\left(K, \mathrm{w}^{*}\right)$ is fragmented by the norm of $X^{*}$.

Now we prove Theorem 7. Observe that we cannot apply Theorem 3 because we do not know whether $\ell_{1}(I)$ has property $J$ when $I$ is uncountable (if $I$ is countable it has because $\ell_{1}(I)$ is separable in this case). In fact, if we assume that there exists an uncountable measurable cardinal $\alpha$ (see $[4$, p. 186, 196] for definitions) and $I$ is a set with $|I|=\alpha$, then it is easy to prove that $\ell_{1}(I)$ fails to have property $J$. 
Proof of Theorem 7. First, observe that $X^{*}=\ell_{\infty}(I)$ and $X^{* *}$ is the space $M_{R}(\beta I)$ of Radon Borel measures on $\beta I$. Thus, $X^{* *}$ has the decomposition

$$
X^{* *}=\ell_{1}(I) \oplus_{1} M_{R}(\beta I \backslash I) .
$$

Notice that the subspace $\ell_{1}(I)$ of this decomposition coincides with the space $J(X), J: X \rightarrow X^{* *}$ being the canonical inclusion. If $\mu \in M_{R}(\beta I)$, we write $\mu=\mu_{1}+\mu_{2}$, where $\mu_{1} \in \ell_{1}(I)$ and $\mu_{2}=\mu_{\uparrow \beta I \backslash I} \in M_{R}(\beta I \backslash I)$. So, $d(\mu, X)=\left\|\mu_{2}\right\|$.

Suppose that there exist a $\mathrm{w}^{*}$-compact subset $K \subset B\left(X^{* *}\right)$ and two numbers $a, b>0$ such that:

$$
d(K, X)<a<b<d\left(\overline{\mathrm{CO}} w^{*}(K), X\right) .
$$

By Lemma 12 we have the following Fact:

Fact. There exist $\psi \in S\left(X^{\perp}\right)$ and a $\mathrm{w}^{*}$-compact subset $\emptyset \neq H \subset K$ such that for every w*-open subset $V$ with $V \cap H \neq \emptyset$ there exists $\xi \in \overline{\mathrm{co}}^{w^{*}}(V \cap H)$ with $\psi(\xi)>b$.

Step 1. By the Fact we can pick $\xi_{1} \in \overline{\mathrm{Co}}^{w^{*}}(H)$ with $\psi\left(\xi_{1}\right)>b$ and $x_{1}^{*} \in S\left(X^{*}\right)$ with $x_{1}^{*}\left(\xi_{1}\right)>b$. Now we choose

$$
\eta_{1}=\sum_{i=1}^{n_{1}} \lambda_{1 i} \eta_{1 i} \in \operatorname{co}(H), \quad \eta_{1 i} \in H, \quad \lambda_{1 i} \geq 0, \quad \sum_{i=1}^{n_{1}} \lambda_{1 i}=1,
$$

such that $x_{1}^{*}\left(\eta_{1}\right)>b$. If $\eta_{1}=\eta_{1}^{1}+\eta_{1}^{2}$, with $\eta_{1}^{1} \in \ell_{1}(I)$ and $\eta_{1}^{2} \in M_{R}(\beta I \backslash I)$, then

$$
\left\|\eta_{1}^{2}\right\|=d\left(\eta_{1}, X\right) \leq d(K, X)<a,
$$

whence $\left\|\eta_{1}^{1}\right\|=\left\|\eta_{1}\right\|-\left\|\eta_{1}^{2}\right\|>b-a$, because $\left\|\eta_{1}\right\| \geq x_{1}^{*}\left(\eta_{1}\right)>b$. So, we can find $y_{1} \in B\left(X^{*}\right)=B\left(\ell_{\infty}\right)$ with finite $\operatorname{support} \operatorname{supp}\left(y_{1}\right)=\left\{\gamma_{11}, \ldots, \gamma_{1 p_{1}}\right\} \subset I$ such that $y_{1}\left(\eta_{1}^{1}\right)>b-a$. Since $y_{1}\left(\eta_{1}^{2}\right)=0$, we have

$$
y_{1}\left(\eta_{1}\right)=y_{1}\left(\eta_{1}^{1}\right)>b-a,
$$

whence it follows that $y_{1}\left(\eta_{1 i}\right)>b-a$ for some $1 \leq i \leq n_{1}$.

Step 2. Let $V_{1}=\left\{u \in X^{* *}: y_{1}(u)>b-a\right\}$, which is a $\mathrm{w}^{*}$-open subset of $X^{* *}$ with $V_{1} \cap H \neq \emptyset$, because $\eta_{1 i} \in V_{1} \cap H$ for some $1 \leq i \leq n_{1}$. By the Fact there exists $\xi_{2} \in \overline{\mathrm{CO}}^{w^{*}}\left(V_{1} \cap H\right)$ with $\psi\left(\xi_{2}\right)>b$. Since $\psi\left(\xi_{2}\right)>b$ and $\psi\left(e_{\gamma_{1 i}}\right)=0,1 \leq i \leq p_{1}$ (where $e_{\gamma_{1 i}} \in \ell_{1}(I)$ is the unit vector such that $e_{\gamma_{1 i}}(\gamma)=1$, if $\gamma=\gamma_{1 i}$, and $e_{\gamma_{1 i}}(\gamma)=0$, if $\left.\gamma \neq \gamma_{1 i}\right)$, there exists $x_{2}^{*} \in B\left(X^{*}\right)$ with $x_{2}^{*}\left(\xi_{2}\right)>b$ and $x_{2}^{*}\left(e_{\gamma_{1 i}}\right)=0,1 \leq i \leq p_{1}$. Clearly, we can choose

$$
\eta_{2}=\sum_{i=1}^{n_{2}} \lambda_{2 i} \eta_{2 i} \in \operatorname{co}\left(V_{1} \cap H\right), \quad \eta_{2 i} \in V_{1} \cap H, \quad \lambda_{2 i} \geq 0, \quad \sum_{i=1}^{n_{2}} \lambda_{2 i}=1,
$$

such that $x_{2}^{*}\left(\eta_{2}\right)>b$. 
As $y_{1}\left(\eta_{2 i}\right)>b-a, 1 \leq i \leq n_{2}$, we get $y_{1}\left(\eta_{2}\right)>b-a$. Let $\eta_{2}=\eta_{2}^{1}+\eta_{2}^{2}$, with $\eta_{2}^{1} \in \ell_{1}(I), \eta_{2}^{2} \in M_{R}(\beta I \backslash I)$ and $\left\|\eta_{2}^{2}\right\|=d\left(\eta_{2}, X\right) \leq d(K, X)<a$. Since

$$
\left\|\eta_{2}^{1}\right\| \geq\left|x_{2}^{*}\left(\eta_{2}^{1}\right)\right|=\left|x_{2}^{*}\left(\eta_{2}\right)-x_{2}^{*}\left(\eta_{2}^{2}\right)\right| \geq\left|x_{2}^{*}\left(\eta_{2}\right)\right|-\left|x_{2}^{*}\left(\eta_{2}^{2}\right)\right|>b-a,
$$

and $x_{2}^{*}=0$ on $\operatorname{supp}\left(y_{1}\right)$, we can find $y_{2} \in B\left(X^{*}\right)$ with finite support $\operatorname{supp}\left(y_{2}\right)=\left\{\gamma_{21}, \ldots, \gamma_{2 p_{2}}\right\} \subset I \backslash \operatorname{supp}\left(y_{1}\right)$ such that $y_{2}\left(\eta_{2}^{1}\right)>b-a$. Hence, $y_{2}\left(\eta_{2}\right)=y_{2}\left(\eta_{2}^{1}\right)>b-a$ and this implies $y_{2}\left(\eta_{2 i}\right)>b-a$ for some $1 \leq i \leq n_{2}$.

By reiteration, we obtain the sequence $\left\{y_{k}\right\}_{k \geq 1} \subset B\left(X^{*}\right)$ with pairwise disjoint supports and the sequence $\left\{\eta_{k}\right\}_{k \geq 1} \subset \operatorname{co}(H) \subset B\left(X^{* *}\right)$ such that $y_{n}\left(\eta_{k}\right)>b-a$ for $k \geq n$.

Since $\left\|\sum_{i=1}^{n} y_{i}\right\| \leq 1$ (because the vectors $\left\{y_{k}\right\}_{k \geq 1} \subset B\left(\ell_{\infty}\right)$ have pairwise disjoint supports) and $\left(\sum_{i=1}^{n} y_{i}\right)\left(\eta_{n}\right)>n(b-a), \forall n \geq 1$, we get $\left\|\eta_{n}\right\|>$ $n(b-a), \forall n \geq 1$, a contradiction, because $\left\|\eta_{n}\right\| \leq 1$.

Acknowledgements. The author would like to thank the referee for many suggestions which helped to improve this paper.

\section{References}

[1] Argyros, S., Mercourakis, S. and Negrepontis, S.: Functionalanalytic properties of Corson-compact spaces. Studia Math. 89 (1988), 197-229.

[2] Argyros, S. And Mercourakis, S.: On weakly Lindelöf Banach spaces. Rocky Mountain J. Math. 23 (1993), 395-446.

[3] Choquet, G.: Lectures on analysis. Vol. II: Representation theory. A. Benjamin, Inc., New York-Amsterdam, 1969.

[4] Comfort, W. W. and Negrepontis, S. A.: Chain Conditions in topology. Cambridge Tracts in Math. 79. Cambridge Univ. Press, 1982.

[5] Diestel, J. And UhL, J. J.: Vector measures. Mathematical Surveys 15. American Mathematical Society, Providence, R.I., 1977.

[6] Fabian, M.: Gâteaux differentiability of convex functions and topology. Weak Asplund Spaces. Canadian Mathematical Society Series of Monographs and Advanced Texts. A Wiley-Interscience Publication. John Wiley \& Sons, Inc., New York, 1997.

[7] Fabian, M., Hájek, P., Montesinos, V. and Zizler, V.: A quantitative version of Krein's Theorem. Rev. Mat. Iberoamericana 21 (2005), no. $1,237-248$.

[8] Fabian, M., Montesinos, V. And Zizler, V.: A characterization of subspaces of weakly compactly generated Banach spaces. J. London Math. Soc. (2) 69 (2004), no. 2, 457-464. 
110 A. S. Granero

[9] Haydon, R.: Some more characterizations of Banach spaces containing $\ell_{1}$. Math. Proc. Cambridge Philos. Soc. 80 (1976), 269-276.

[10] NamiokA, I.: Radon-Nikodým compacts spaces and fragmentability. Mathematika 34 (1987), no. 2, 258-281.

[11] Negrepontis, S.: Banach spaces and topology. In Handbook of settheoretic topology, 1045-1142. North-Holland, 1984.

Recibido: 6 de febrero de 2003

Revisado: 30 de abril de 2004

Antonio S. Granero

Departamento de Análisis Matemático

Facultad de Matemáticas

Universidad Complutense de Madrid

28040-Madrid, Spain

AS_granero@mat.ucm.es

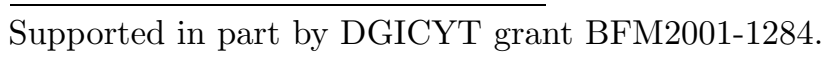

